\title{
De las perspectivas provisorias de Friedrich Nietzsche a los fundamentos contingentes de Judith Butler ${ }^{*}$
}

* Fecha de recepción: 6 de julio de 2015. Fecha de aceptación: 15 de marzo de 2016
Malena Nijensohn**

\section{Resumen}

En el presente artículo ${ }^{1}$ me propongo analizar la noción de 'fundamentos contingentes' de Judith Butler y ponerla en relación con la idea de las "perspectivas provisorias" en la filosofía de Friedrich Nietzsche. Considero que la crítica nietzscheana a la metafísica de la sustancia ofrece la (des)base ontológica para las reflexiones ético-políticas del siglo XX, en las cuales se inserta Butler. Así, la "muerte de Dios", es decir, la crítica a las nociones de 'fundamento' y 'verdad absoluta', se muestra como el marco teórico propicio para los desarrollos posfundacionalistas de Butler. Abordaré entonces, en primer lugar, la crítica de Nietzsche al pensamiento tradicional occidental. En segundo lugar, analizaré la diferenciación que propone Butler entre posmodernidad y posfundacionalismo, de forma tal que quede delineada su teoría y su propuesta de pensar en 'fundamentos contingentes'.

\footnotetext{
Abstract

In this paper I will analyze the concept by Judith Butler 'contingent foundations', to connect it with the idea of 'provisional perspectives' in Friedrich Nietzsche's philosophy. I consider that Nietzsche's criticism of the metaphysics of substance is the ontological basis to ethic and political reflections in the $20^{\text {th }}$ Century, in which Butler is inserted. Thus, "Death of God", which is the critique of 'foundation' and 'absolute truth', is the theoretical frame propitious for the post-foundational developments by Butler. I will first address the critique by Nietzsche to the traditional occidental thinking. I will then analyze the difference proposed by Butler between postmodernism and post-foundationalism, to delineate her theory and her proposal of thinking about 'contingent foundations'.
}

\section{Palabras clave:}

Perspectivas provisorias, muerte de Dios, fundamentos contingentes, posmodernidad, posfundacionalismo

1 Este trabajo forma parte de mi proyecto de doctorado titulado "Poder y resistencia: la (de)construcción de y resistencia: la (de)construcción de
las subjetividades sexo-generizadas. Una lectura sobre la teoría de Judith Butler", radicado en el Instituto Interdisciplinario de Estudios de Género de la FFyL de la UBA, dirigido por la Dra. Virginia M. Cano.

\section{Key words:}

Provisional perspectives, death of God, contingent foundations, postmodernism , post-foundationalism 
2 Traduzco de aquí en más el concepto de 'postmodernism' por 'posmodernidad'.
3 Sigo aquí la lectura de Burgos Díaz, que sostiene que Butler y Nietzsche conforman una comunidad intelectual a través de las mediaciones de Foucault y Derrida. Cfr. "Desde el marco postestructuralista en el que se sitúa, Butler reconoce explícita-

se situa, Butler reconoce explícitade Derrida; autores que, como ellos mismos afirman, son deudores de esa inicial crítica a la tradición occidental inaugurada por Nietzsche. Desde este ángulo, es claro que hay una cierta comunidad intelectual desde Nietzsche hasta Butler" (Burgos Díaz, 2008: 157).
En el artículo titulado "Fundamentos contingentes: el feminismo y la cuestión del 'postmodernismo'" (Butler, 2001), ${ }^{2}$ Butler toma como punto de partida una crítica que se le ha hecho a las teorías posmodernas, a saber: que al presentar como contingentes ciertos conceptos antes tematizados como fundacionales, abandonan sus compromisos políticos. Pareciera haber, desde esta perspectiva, una oposición radical entre lo fundante y lo contingente y, en el interior de dicha oposición, la política quedaría siempre del lado de lo fundacional. Como la posmodernidad sostiene que no hay fundamentos estáticos e inamovibles, queda entonces indefectiblemente incapacitada para reflexionar sobre las cuestiones políticas que, siempre según esta crítica, se basan en un fundamento (por lo general, considerado 'último').

Nuestra autora se encarga, sin embargo, de poner el signo de interrogación sobre esta crítica y de dislocar la oposición dicotómica entre fundamento y contingencia. De la proposición que sostiene que no hay un fundamento último, ¿se sigue necesariamente que no hay ningún suelo desde el cual pensar la política? Para mostrar justamente lo contrario, Butler recurre al término 'posfundacional' y lo diferencia de la 'posmodernidad'.

Ahora bien, querría centrarme en una problemática previa y que considero menester desarrollar para entender la posición teórica de Judith Butler en este punto. Dicha cuestión pretende interrogar un antecedente que es, desde mi perspectiva, clave para comprender los postulados posmodernos y, sobre todo, la posición posfundacionalista de la norteamericana. Me refiero a la filosofía de Friedrich Nietzsche, considerada como un punto de inflexión en la historia del pensamiento de la metafísica occidental. ${ }^{3}$ En este artículo me abocaré entonces a las siguientes problemáticas: ¿en qué sentido se puede pensar que la filosofía de Friedrich Nietzsche es un antecedente del pensamiento posfundacionalista de Judith Butler? ¿En qué se distancia Butler de la posmodernidad, para ubicarse en la línea que ella denomina 'posfundacionalista'? ¿Qué consecuencias se pueden extraer a nivel de la práctica política?

Sostengo la hipótesis de que indagar la crítica de Nietzsche a la noción de 'fundamento' puede echar luz sobre la conceptualización del 'posfundacionalismo' adoptada, entre otros, por Butler. A partir de las críticas nietzscheanas a la metafísica de la sustancia y su consecuente propuesta de una ontología no esencialista, se abre el marco contemporáneo en el cual son posibles las preguntas ético-políticas que desarrolla Butler.

En primer lugar, abordaré la crítica de Nietzsche a la noción occidental tradicional de 'fundamento'. Su proclama de la "muerte de Dios" y la consiguiente apertura de un espacio para la filosofía de la creación se revelarán como el marco teórico propicio para el posfundacionalismo en el cual se encuadra Judith Butler. En segundo lugar, analizaré entonces la diferenciación entre posmodernidad y posfundacionalismo, de forma tal que quede delineada la teoría butleriana y su propuesta de pensar en 'fundamentos contingentes'.

\section{La crítica de Friedrich Nietzsche a la noción de fundamento}

“Que el valor del mundo reside en nuestra interpretación -que quizá en alguna parte sean posible otras interpretaciones, diferentes de las meramente humanas-, que las interpretaciones habidas hasta ahora son estimaciones perspectivistas en virtud de las cuales nos mantenemos en vida, es decir, en la 
voluntad de poder, de crecimiento del poder, que toda elevación del hombre lleva consigo la superación de interpretaciones más estrechas, que toda fortificación y ampliación de poder que se alcance abre nuevas perspectivas y hace creer en nuevos horizontes - esto recorre mis escritos".

\section{Friedrich Nietzsche, Fragmentos póstumos}

En esta sección abordaré la crítica efectuada por el filósofo alemán Friedrich Nietzsche a la metafísica de la sustancia, dado que ella ofrece el marco general de análisis para deconstruir la noción de 'fundamento' que las filosofías tradicionales de Occidente han forjado y, por tanto, se revela como uno de los pilares sobre los que Butler asienta sus tesis posfundacionalistas. Con la noción de 'metafísica de la sustancia' me refiero a la Weltanschauung occidental tradicional que ha establecido los esquemas conceptuales a través de los cuales se ha regido la historia de los hombres. Dicha cosmovisión ha postulado siempre la creencia en un fundamento último y en una verdad absoluta. ${ }^{4}$

Hay dos grandes críticas generales que abarcan, desde la perspectiva de Nietzsche, a todos los pensadores de la tradición occidental. A modo de nota preliminar: como se sabe, las apreciaciones de la tradición occidental efectuadas por Nietzsche de ningún modo les hacen justicia a los autores trabajados, pues el propósito nietzscheano no es efectuar una reconstrucción histórica fiel, sino, muy por el contrario, destacar ciertos puntos que a él le parecen centrales y le permiten confeccionar la historia del nihilismo decadente. En el apartado "La 'razón' en la filosofía", en el Crepúsculo de los idolos (Nietzsche, 2006: 51-56), Nietzsche destaca dos idiosincrasias constitutivas de todos los filósofos: en primer lugar, la falta de sentido histórico y, en segundo lugar, la confusión de lo último con lo primero. La primera idiosincrasia señalada -la falta de sentido histórico - remite a que los filósofos no reconocen que los conceptos son creaciones humanas, demasiado humanas y que, en cuanto tales, tienen una historia y pueden asimismo ser destruidos. Según Nietzsche, estos filósofos parten de una dicotomía excluyente entre el ser y el devenir y optan por el ser, considerando el devenir como algo despreciable e indigno de veneración (Nietzsche, 2006: 51). Creen que honrar algo es deshistorizarlo y considerarlo eterno, y que la procreación, el cambio y la muerte son refutaciones y objeciones. De allí que intenten por todos los medios sobreimprimirle el sello del ser al devenir (Nietzsche, 2006: 52). Nietzsche llama a esta actitud "egipticismo", aludiendo a la voluntad de petrificación y permanencia estática y a la construcción de monumentos (Nietzsche, 2006: 51). "Todo lo que los filósofos han venido manejando desde hace milenios fueron momias conceptuales; de sus manos no salió vivo nada real" (Nietzsche, 2006: 51), sostiene. De este modo, los filósofos se convierten en sepultureros, y la filosofía, en la representación del monótono-teismo: un pensamiento monótono respecto del theós; es decir, un theós que se piensa como idéntico a sí mismo, que representa la mismidad y la identidad. ${ }^{5}$

La segunda idiosincrasia de todos los filósofos consiste, siempre según Nietzsche, en "confundir lo último con lo primero". Es decir, olvidando que han sido ellos mismos quienes han inventado este modo de ser de las cosas, pretenden que estas tengan un origen en sí mismas (Nietzsche, 2006: 53). Siguiendo la línea de pensamiento de Nietzsche, los filósofos creen que las cosas se les aparecen como son en sí mismas, y no como ellos las han interpretado o diseñado. Sostienen, además, que ninguno de los conceptos supremos puede tener una historia, puede haber devenido: con esto, lo último (aquello inventado por "estos tejedores de telarañas", al decir de nuestro filósofo) tiene que ser lo primero, tiene que ser causa sui (Nietzsche, 2006a: 53). Bajo este prejuicio, advierte Nietzsche, los metafísicos no pueden concebir cómo podría una cosa surgir de su antítesis y consideran que las cosas de valor deben tener un
${ }_{4}$ Cfr. "Esta crítica [la crítica nietzscheana a la metafísica] apunta a la deconstrucción de los conceptos que se organizan en un sistema piramidal centrado en torno a un principio que opera como arkhé-dios fundamento del ser, del pensar y del obrar" (Cragnolini, 2006: 27-28).

5 Cfr. "El término creado por Nietzsche, Monotono-theismus, resulta muy sugerente para caracterizar los sistemas metafísicos que surgen a partir de una ilusión óptico-moral. La metafísica genera conceptos atravesados por la inmutabilidad (egipticismo) que se organizan sistemático-piramidalmente a partir de un principio que opera como arkhé, elemento jerarquiopera como arkhe, elemento jerarquiy del obrar" (Cragnolini, 2003: 61). 
6 Como señala A. Sánchez Pascual en su traducción a Crepúsculo de los ídolos, la Froschperspektive es la “visión de un objeto desde un punto de vista situado por debajo de él; todo objeto situado por encima de la 'altura del ojo' se dice que es mirado con 'perspectiva de rana'. Por extensión, este término significa también en alemán: modo banal y corriente de pensar" (Nietzsche, 2000: 277). 7 Cfr. "Pese a todo el valor que "Pese a todo el valor que a lo veraz, a lo desinteresado: sería posible que a la apariencia, a la voluntad de engaño, al egoísmo y a la concupiscencia hubiera que atribuirles un valor más elevado o más fundamental para toda vida Sería incluso posible que lo que constituye cluso posible que lo que constituye el valor de aquellas cosas buenas y
veneradas consistiese precisamente en el hecho de hallarse emparentadas, vinculadas, entreveradas de manera capciosa con estas cosas malas, aparentemente antitéticas, y quizás en ser idénticas esencialmente a ellas" (Nietzsche, 2000: 24).

8 Cfr. "La pseudoverdad es aquella que estatiza lo deviniente pero de que: congelándolo estructuras que se autopostulan como eternas, apriorísticas, necesarias" (Cragnolini, 2003: 74). origen propio, pues no pueden brotar de lo pasajero y de lo engañoso. Concluyen entonces que su fundamento se halla en el Ser. Para Nietzsche, estos prejuicios de los filósofos, que se encuentran en la base de todos sus procedimientos lógicos, corresponden a "una perspectiva de rana" (Froschperspektive), esto es, la perspectiva sesgada de quien mira de abajo hacia arriba. ${ }^{6}$ Según el filósofo alemán, plantear el pensamiento en estos términos binarios y antitéticos, valorando un extremo y despreciando el otro, implica una pérdida de los matices, impidiéndole así a la filosofía entrever la articulación entre los dos polos, articulación en la cual, según advierte, quizás radique el valor tanto de un término como del otro.

En esta misma línea, Nietzsche concluye que cuando los filósofos se jactan de sus juicios, aduciendo que son verdaderos, no hacen sino olvidar que fueron ellos mismos quienes los inventaron. Pues bien, hemos visto que los metafísicos se han encargado de poner el Ser allí donde había devenir y que tienen además una tendencia a confundir lo último con lo primero; por tanto, quizás sea este el punto donde se aúnan las dos idiosincrasias de los filósofos, a saber: en que interpretan creando un mundo calculable, simplificado y comprensible, y luego olvidan que son ellos mismos quienes lo han inventado de este modo, razón por la cual pretenden que el mundo se les presente objetivamente. Pero los hechos puros, en sí, no existen para nuestro filósofo. "No se vuelve a encontrar en las cosas nada que uno mismo no haya introducido en ellas", sentencia lacónicamente (Nietzsche, 2006b: 130).

En suma, para el filósofo alemán "no hay hechos, solo interpretaciones" (Nietzsche, 2006b: 222), y esto quiere decir que todo Ser es serinterpretado, que no hay una forma en la que las cosas sean por fuera del marco interpretativo. En contra de la tradición que postula dos planos separados, uno ontológico y otro lingüístico, que pueden corresponderse generando juicios verdaderos o pueden no corresponderse generando juicios falsos, Nietzsche desarticula tal separación, pues no hay "cosas en sí" a las que el lenguaje haga referencia, sino que la ontología misma está constituida discursivamente. En otras palabras, las interpretaciones y las perspectivas constituyen lo que es, dan sentido y configuran el devenir. Este giro nietzscheano es clave para comprender la línea filosófica en la cual se inserta Butler: aquí radica el núcleo que rompe con la noción moderna de 'fundamento'.

Nietzsche propone no seguir pensando en términos de juicios verdaderos o falsos, pues tal distinción no tiene sentido luego de estas consideraciones, sino, por el contrario, en términos perspectivistas. Cada juicio, cada valoración, cada modo de ver constituye una perspectiva que organiza y determina una forma de vida. No se trata ya de totalidades verdaderas que se podrían alcanzar con un buen método, sino de ordenaciones de sentido que permiten sistematizar el caos, siempre provisoriamente. Uno de los problemas principales que el filósofo alemán encuentra en la historia de la metafísica es que esta ha considerado sus juicios como verdades encontradas, "descubiertas" (aquí, según cada tradición, la metodología utilizada ha sido diferente, pero el punto central es que siempre se ha creído que podía hallarse la "Verdad"). Los filósofos no han sabido reconocer el carácter ficcional de su Verdad. Lo que han creado no han sido sino pseudoverdades; es decir, juicios que se toman por verdaderos sin reconocer su carácter producido e histórico. ${ }^{8}$

Que no haya ninguna verdad no significa, sin embargo, que tengamos que vivir sin ella. Nietzsche dice dos cosas que, aunque parezcan contradictorias, se las comprende perfectamente cuando están articuladas: por un lado, "que no hay verdad, que no hay constitución absoluta de las cosas, que no hay 'cosa en sí'” (Nietzsche, 2006b: 242); por el otro, que "es necesario que algo tenga que ser tomado por verdadero" (Nietzsche, 2006b: 243). Aquí cabría distinguir entre dos sentidos de verdad: en primer lugar, se la entiende en términos de una verdad última y absoluta que, como hemos mostrado, 
es menester que sea descartada. En segundo lugar, la verdad aparece como una perspectiva provisoria configuradora de sentido. En cuanto tal, es una ficción necesaria para la vida. En palabras del propio Nietzsche (2000:25-26):

La falsedad de un juicio no es para nosotros ya una objeción contra él; acaso sea en esto en lo que más extraño suene nuestro nuevo lenguaje. La cuestión está en saber hasta qué punto ese juicio favorece la vida, conserva la vida, conserva la especie, quizá incluso selecciona la especie; y nosotros estamos inclinados por principio a afirmar que los juicios más falsos [...] son los más imprescindibles para nosotros, que el hombre no podría vivir si no admitiese las ficciones lógicas, si no midiese la realidad con el metro del mundo puramente inventado de lo incondicionado, idéntico-a-sí-mismo, si no falsease permanentemente el mundo mediante el número, que renunciar a los juicios falsos sería renunciar a la vida, negar la vida.

Es decir, Nietzsche sostiene, entonces, tanto la inexistencia de un concepto absoluto de verdad como la necesidad de crear alguna verdad, es decir, de instituir valores. Nuevamente en los términos de nuestro filósofo (Nietzsche, 2006b:246):

[E]l constatar entre "verdadero" y "no verdadero", el constatar en general situaciones de hecho, es fundamentalmente diferente del poner creativo, del configurar, conformar, subyugar, querer, tal como se encuentra en la esencia de la filosofía. INTRODUCIR UN SENTIDO - esta tarea sigue absolutamente pendiente, puesto que no hay allí ningún sentido.

En este fragmento póstumo, Nietzsche señala una diferencia esencial entre la constatación y la creación, pues del primer modo caemos en el nihilismo decadente y del segundo, por el contrario, llegamos a la filosofía del futuro. Es decir, según el primero, desembocamos en la creencia de que hay algo verdadero y algo no verdadero que nosotros debemos encontrar. Sin embargo, cuando se trata de la tarea creativa - como sucede en el segundo caso- entra en juego la ficción y ya no se trata de buscar sino de "crear" la verdad. Esta nueva noción de verdad ya no se considera a sí misma absoluta y ahistórica, sino que asume su carácter finito y ficcional. La tarea de creación de la verdad, de introducción de un sentido, solo puede venir luego de que se haya "aniquilado" (vernichten) la noción de Verdad última. Ahora se ha abierto el terreno del perspectivismo, terreno que antes se encontraba clausurado por verdades pretendidamente absolutas. No se trata ya de buscar un sentido que esté dado en las cosas, sino de introducirlo asumiendo la tarea artística de la creación de perspectivas siempre provisorias.

Sin duda, la noción nietzscheana de 'perspectiva provisoria' arroja luz sobre el concepto butleriano de 'fundamento contingente'. Pero para sostener esta tesis es menester revisar el recorrido que efectúa Judith Butler, tarea a la que me aboco en la siguiente sección de este trabajo.

\section{Posmodernidad, posfundacionalismo y feminismo en Judith Butler}

\section{Respuesta de Butler a las críticas a la posmodernidad}

En el artículo "Fundamentos contingentes: el feminismo y la cuestión del 'posmodernismo"' ya mencionado, Judith Butler se pregunta si existe algo llamado 'posmodernidad" "¿Es una caracterización histórica, un cierto tipo de posición teórica, y qué 
9 Butler, siguiendo a Agnes Heller, Chantal Mouffe y Ernesto Laclau, entre otros teóricos políticos, distingue entre "la política" y "lo político" (Marchart, 2009: 13-14).
10 Cfr. "Si el postmodernismo como término tiene alguna fuerza o significado dentro de la teoría social, o la teoría social feminista social, o la teoría social feminista
en particular, tal vez este puede encontrarse en el ejercicio crítico que muestra cómo la teoría, como la filosofía, está siempre implicada en el poder [...]" (Butler, 2001, 14). significa para un término que ha descrito una cierta práctica estética ser aplicado ahora a la teoría social, y en particular a la teoría social y política feminista?" (Butler, 2001: 8).

Aquí surgen dos cuestiones: en primer lugar, que los así llamados "posmodernos" no se han dado a sí mismos ese nombre, sino que son otros autores, críticos de esta corriente de pensamiento, los que así los han nombrado para unificarlos bajo una misma categoría de pensamiento. La segunda cuestión es, ¿qué autores han sido considerados "posmodernos"? Y aquí, Butler sostiene que se trata de aquellos que han realizado o bien una crítica del sujeto, o bien un análisis discursivo, es decir, aquellos que han cuestionado la integridad o la coherencia de descripciones sociales totalizantes. En suma, serían todos los autores que han planteado la pérdida de un fundamento último que estructure la realidad y, en consecuencia, modifiquen nuestra comprensión de ella.

Butler reconoce que esto bien podría derivar en un nihilismo absoluto según el cual no habría ningún locus desde el cual reflexionar sobre la política. Esta es, de hecho, la crítica que ofrecen los detractores de la posmodernidad: si no hay un fundamento último, un punto árkhico que funde la política, la ética o el pensamiento, entonces "todo vale" o bien "nada vale", es decir, se puede pensar cualquier cosa o no se puede pensar en absoluto.

Contrariamente, según Butler reconoce, las posiciones fundacionalistas, que sostienen que la política es impensable sin un fundamento último, son precisamente posiciones autoritarias que, al contrario de lo que pretenden, clausuran el dominio de lo político. Si se establece un principio como inamovible e indiscutible, ¿dónde queda entonces la discusión sobre lo político? ${ }^{9}$ Justamente, al contrario de lo que plantean los detractores de la posmodernidad, para habilitar el campo de lo político es necesario que este sea un espacio abierto en el cual se puedan disputar los sentidos establecidos. "El acto que establece unilateralmente el dominio de lo político funciona entonces como un subterfugio autoritario mediante el cual la disputa política sobre el estatus del sujeto es silenciada sumariamente" (Butler, 2001: 9).

Así, esta filósofa muestra la paradoja en la que quedan atrapados los fundacionalistas críticos de la posmodernidad: en efecto, en su afán por caracterizar un conjunto de teorías y agruparlas a todas bajo el mismo signo, convierten la posmodernidad en una época, en un todo sintético y mezclan toda una serie de teorías tales como el posestructuralismo, la deconstrucción, el feminismo francés, el psicoanálisis lacaniano, el análisis foucaultiano, el conversacionalismo de Rorty, los estudios culturales, en un todo único. En este sentido, las consideran modalidades o permutaciones subordinadas bajo un mismo sustantivo. Es decir, a juicio de Butler, subyugan la diversidad y la diferencia bajo un único rótulo conceptual. En su supuesto intento por democratizar la discusión política, lo que hacen, finalmente, podría caracterizarse - sostiene Butler - como una acción autoritaria cuyo efecto es el contrario de las intenciones manifiestas. En las palabras de nuestra teórica: “Es, en el mejor de los casos, paradójico que el acto de maestría conceptual que lleva a cabo esta desdeñosa agrupación de posiciones bajo el postmodernismo, quiera hacer a un lado el peligro del autoritarismo político" (Butler, 2001: 13).

Así, los detractores de la posmodernidad consideran que las teorías son totalidades organizadas que, bajo el signo hegeliano, emergen como la articulación de una condición humana específica que se desenvuelve históricamente. Pero justamente esto es lo que ha sido disputado por las teorías ahora unificadas como posmodernas. Si hay algo que pueda unirlas de alguna forma es, justamente, que en ellas hallamos un ejercicio crítico que devela que toda posición teórica o filosófica implica siempre una operación de poder. ${ }^{10}$ Cuando las teorías se pretenden por fuera de dicho juego 
de poder, lo que hacen a fin de cuentas es utilizar una estratagema de poder que las legitima, pero que queda encubierta. Así, buscan las supuestas bases metapolíticas, unos fundamentos en apariencia inconmovibles que universalizan y naturalizan.

\section{No hay fundamento, necesitamos fundamentos}

Hasta el momento hemos planteado la cuestión de forma dicotómica: o bien fundacionalismo o bien antifundacionalismo. Si el debate quedara planteado en estos términos, nos encontraríamos frente a una aporía. El fundacionalismo supone que la política se basa en principios universales que no son pasibles de revisión y que fundan el ámbito de lo político. Por su parte, el antifundacionalismo sostiene que no hay ningún fundamento absoluto. Como sostiene Marchart (2009: 26-27):

... en la medida en que la visión antifundacionalista se basa en la negación o en la oposición respecto de la visión fundacionalista, obviamente comparten el mismo horizonte. [...] De acuerdo con los primeros [los fundacionalistas], los antifundacionalistas necesariamente tienen que valerse del fundacionalismo para desarrollar el antifundacionalismo; además, al invalidar todos los fundamentos, están erigiendo, en rigor, un nuevo fundamento final, una suerte de "antifundamento". Por lo tanto, deberían aceptar que es finalmente imposible superar el fundacionalismo.

Así, Marchart muestra cómo el antifundacionalismo también se ve obligado a recurrir a un fundamento, por más de que este sea, por definición, un "antifundamento", el fundamento que sostiene que no hay fundamento. Tanto el fundacionalismo como el antifundacionalismo quedan enmarcados en el paradigma hegemónico fundacionalista, que impone los términos de la discusión y obliga al antifundacionalismo a ingresar en su territorio, aunque este último no pretenda sino plantearse por fuera de dicho paradigma. Butler (2001:15) examina este desafío:

No sé en el caso del término "postmoderno", pero sí hay un punto, y un punto muy fino, en lo que yo quizá entiendo mejor como postestructuralismo, ese es que el poder contamina el propio aparato conceptual que busca negociar sus términos, incluyendo la posición del crítico, y más aún, que esta implicación de los términos de la crítica en el campo del poder no es el advenimiento de un relativismo nihilista incapaz de crear normas, sino más bien la misma precondición de una crítica políticamente comprometida.

Planteada de este modo la cuestión, si la posmodernidad cae en un nihilismo y las posiciones críticas de la posmodernidad caen en un autoritarismo, entonces nos encontramos frente a una encrucijada sin salida donde no hay posibilidad para lo político. Por un lado, si se asumiera una posición antifundacionalista, si se intentara deshacerse de los fundamentos, entonces no habría ningún suelo desde el cual efectuar la práctica o la reflexión políticas. Por otro, si se adoptara, por el contrario, una posición que estableciera ciertas normas más allá del poder a través de figuras retóricas de universalidad, entonces no habría discusión posible: el autoritarismo cancelaría la práctica y la reflexión políticas. Para Butler, estas dos posiciones van juntas como diferentes versiones del fundacionalismo y de la problemática escéptica que este engendra. En efecto, la dicotomía fundacionalismo-antifundacionalismo implica siempre darle un fundamento último a lo político, ya sea bajo la forma positiva que le otorga algún contenido o bajo la forma negativa que niega que exista un fundamento.

Ahora bien, si nos posicionamos en el polo fundacionalista, tenemos que erigir algún punto árkhico que fundamente todo el ámbito de lo político pero, en este caso, ¿qué discusión política sería posible? ¿Qué cambios podrían introducirse? Como he desarrollado en la sección sobre Nietzsche, sostengo que cuando se plantea una verdad 
11 En este sentido, se podría pensar a Butler como heredera de la forma de filosofar nietzscheana, que no se limita a una mera destrucción negativa de un polo dicotómico (en este caso, la destrucción de la noción de 'fundamento'), sino que sostiene que el pensar es siempre tensionante, que la negación-destrucción y la afirmación-construcción están siempre en tensión. Por ello retomo la interpretación de Mónica Cragnolini y su concepto de "Zwischen" ("entre") a partir del cual ella lee la tarea a f́cica propuesta por Nietzsche.

Cfr. "Una de las cara por Nietzsche.

acterísticas del

modo de filosofar de Nietzsche que

permite la apertura a otras formas de entender el pensamiento es el aspecto tensional de su filosofía, esa presencia de la negación y de la afirmación que no se resuelve en una síntesis. Al Nis se rútic

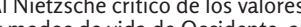
y modos de vida de Occidente, a destructor de las grandes nociones que conforman las arquitecturas filosóficas y morales, al negador y maestro de la sospecha, no le 'sigue' el Nietzsche afirmador y creador de nuevos sentidos, como si una 'evolución' del pensamiento obligara

firmar después de negar, a construir después de destruir, olvidando el
dirmar después de negar, a construir oder de la negación. No existe superación del 'no' por el 'sí', sino que tal vez lo característico del pensamiento nietzscheano sea justamente e mantenimiento de la tensión entre el sí y el no" (Cragnolini, 2006: 27). 12 Cfr. “.... cualquier concepto totalizador de lo universal suprimirá en vez de autorizar los reclamos no previstos ni previsibles que serán hechos bajo el signo de lo 'universal'.

En este sentido, no estoy acabando con la categoría sino tratando de 作 ceso fundamentalista para convertirla en un sitio de disputa política permanente" (Butler, 2001: 18). última no hay lugar para el cambio, el devenir ni la transformación, pues se genera una suerte de sistema deductivo ineludible. Ahora bien, si nos posicionamos en el polo opuesto antifundacionalista, tendremos que sostener que no hay nada a partir de lo cual podamos pensar, discutir o efectuar la práctica de lo político. Esto nos llevaría a un nihilismo extremo que nos paralizaría.

Pareciera, entonces, menester deshacerse de esta dicotomía excluyente y no situarse en ninguno de sus dos extremos sino entre ellos. ${ }^{11}$ Como sostiene Judith Butler (2001: 16), hay que cuestionar lo que el movimiento teórico que establece los fundamentos autoriza y lo que excluye. En sus palabras:

Parece que la teoría postula fundamentos incesantemente, y forma compromisos metafísicos implícitos como si nada, aun cuando busca proteger en contra de ello: los fundamentos funcionan como lo incuestionado y lo incuestionable dentro de cualquier teoría. Sin embargo, ¿no son estos “fundamentos”, aquellas premisas que funcionan como bases autorizadas, constituidos mediante exclusiones que, tomadas en cuenta, exponen la premisa fundamental como una presunción contingente y disputable?

Butler propone realizar no una mera inversión de los términos fundacionalistas, sino una subversión del terreno donde opera el fundacionalismo y una deconstrucción de sus premisas. Por eso introduce la noción de 'fundamentos contingentes', que debilita el estatus ontológico del fundamento sin por eso suprimirlo por completo. No se supone así la ausencia de cualquier fundamento, sino la de un fundamento último. ${ }^{12} \mathrm{El}$ posfundacionalismo butleriano propone, en efecto, la ausencia de un fundamento final, pero no se detiene ahí y por ello no se transforma ni en un mero nihilismo ni en un pluralismo posmoderno. Por el contrario, acepta la necesidad de algunos fundamentos contingentes.

¿Podríamos, entonces, decir que las teorías "posfundacionalistas" son aquellas que se inscriben en una línea posnietzscheana de pensamiento? De ser así, el posfundacionalismo incluiría las filosofías que, tras la "muerte de Dios", postulan que no hay fundamentos últimos, puntos árkhicos del pensamiento a partir de los cuales sostener los edificios teóricos. Sin embargo, tal como ha quedado puesto en evidencia con los desarrollos de la filosofía nietzscheana, no hay fundamentos últimos aunque los fundamentos sean necesarios. Butler aporta su solución a la aporía. Por mi parte, propongo pensar el conflicto social y político como el enfrentamiento de los diversos fundamentos y, de esta forma, los fundamentos contingentes se manifestarían como el locus de una insistente disputa y resignificación agónica, para usar un concepto de Chantal Mouffe (Mouffe, 2013: 16 y ss.). Esto evitaría tanto el autoritarismo, que impide el cambio social o político, como la imposibilidad del cambio. Porque si una situación política estuviera dada de forma universal, no habría posibilidad de modificar su sistema de inclusión o exclusión. De este modo, también se evitaría el pluralismo relativista según el cual todas las posiciones son posibles y pueden convivir en armonía porque de todas formas no hay ninguna base desde la cual juzgar o valorar, lo que nos lleva a un quietismo que, una vez más, imposibilitaría el cambio social o político. Lo interesante del planteo de Judith Butler es que ella no acaba con la noción de fundamento, sino que la aliviana de su peso ontológico sin desconocerlo por completo, para que se convierta en el sitio de disputa permanente de lo político. 


\section{Conclusiones}

Querría volver, para concluir, sobre algunas de las cuestiones iniciales que dispararon este trabajo. La crítica nietzscheana a la metafísica de la sustancia se mostró como la (des)base ontológica que, dos siglos más tarde, operó como el marco teórico de las teorías contemporáneas en las cuales, entre otros, se inserta Judith Butler. Dicha crítica se ha manifestado como un antecedente capaz de echar luz sobre los planteos de la estadounidense. Ambos autores sostienen tanto la inexistencia de fundamentos últimos universales como la necesidad de crear, en los términos de Nietzsche, perspectivas provisorias que, en su terminología conceptual, Butler denomina 'fundamentos contingentes'.

Es decir que ni el alemán ni la estadounidense repudian, sin más, los conceptos que se proponen como el suelo a partir del cual pensar lo político. Lo que ambos autores se proponen es criticar que dichos conceptos funcionen como premisas incuestionables, que se hallen más allá del poder y que, en cuanto supuesto ontológico último, no puedan ser modificados. Plantear fundamentos universales sin más no es sino, a los ojos de ambos autores, una estrategia que disimula su propio juego de poder. Lo que se proponen, entonces, es analizar los procesos de construcción de dichos conceptos, los mecanismos de poder que operan y las implicancias políticas que conllevan. Esto abre el campo de la discusión y de las luchas en torno a lo político, y es por ello que dichos conceptos pueden ser pensados como fundamentos, sí, pero contingentes, provisorios, transitorios; es decir, subsidiarios de los juegos del poder. 


\section{Q Bibliografía}

"Burgos Díaz, E. (2008). Qué cuenta como una vida. La pregunta por la libertad en Judith Butler. Madrid: Antonio Machado.

"Butler, J. (1992). Contingent Foundations: Feminism and the Question of ,Postmodernism'. En Butler, J. y Scott, J. W. Feminists Theorize the Political. Nueva York, Routledge.

"------ (2001). Fundamentos contingentes: el feminismo y la cuestión del 'postmodernismo'. En La Ventana, núm. 13, trad. M. Silva, Universidad de Guadalajara.

»Cragnolini, M. (2003). Nietzsche. Camino y demora. Buenos Aires, Biblos.

»--------- (2006). Moradas nietzscheanas. Del sí mismo, del otro y del "entre". Buenos Aires, La Cebra.

»Marchart, O. (2009). El pensamiento político posfundacional. La diferencia política en Nancy, Lefort, Badiou y Laclau. Buenos Aires, Fondo de Cultura Económica.

»Mouffe, C. (2013). Agonística. Pensar el mundo políticamente. Buenos Aires, Fondo de Cultura Económica.

"Nietzsche, F. (1980), Sämtliche Werke. Kritische Studienausgabe in 15 Bänden, Herausgegeben von G. Colli und M. Montinari, Berlin, Walter de Gruyter/ Deutsche Tachenbuch Verlag.

»---------- (2000). Más allá del bien y del mal, trad. A. Sánchez Pascual, Madrid, Alianza.

»-------- (2006a). Crepúsculo de los ídolos, trad. A. Sánchez Pascual. Madrid, Alianza.

»---------- (2006b). Fragmentos póstumos, vol. IV (1885-1889), edición española dirigida por Diego Sánchez Meca, trad., introducción y notas de J. L. Vermal y J. B. Llinares. Tecnos. 